\title{
3 Methodologie
}

Die zehn hier systematisch analysierten Museen wurden im Kontext der Transformationsprozesse nach 1989, des europäischen Einigungsprozesses sowie der jeweiligen nationalen Geschichtspolitik und Museumslandschaft untersucht. Kombiniert werden die von Museumsstudien (MacDonald 2006; Baur 2013; Heesen 2012; Byrne et al. 2011; Muttenthaler und Wonisch 2006, 37; Bal 1996; Rüsen, Ernst und Grütter 1988) entwickelte site analysis, Diskursanalyse (Keller 2011; Jäger 2004; Titscher, Meyer, Vetter und Wodak 2000), visual history (Paul 2013; Hansen-Gluecklich 2010; Brink 2008; Brink und Wegerer 2012; Knoch 2001; Bredekamp 2004; Korff 1999) und - die ,traditionelle‘ Methodik verlassend - die Analyse von Museen als hybride Medien, die im Zusammenspiel der verschiedenen Medien eine über die einzelnen Elemente hinausgehende Bedeutung entfalten. ${ }^{34}$ Mit Mieke Bal wird gefragt: „How do, far from being opposite or even distant domains, political work and aesthetic work operate together in an inextricable merging that strengthens both?“ (Bal 2007, 16) ${ }^{35}$ Die Untersuchung fand zunächst für jedes Museum gesondert statt und zwar jeweils auf drei Ebenen (Crane 2000, 2):

1. Raum und seine Kodifizierung: Der Ort und die Rolle des Museums

2. Leitnarrativ und Storyline

3. Ästhetik, 3D-Objekte, Visuelles und Text

34 Problematisch erweist sich hingegen die Analyse bloß einer Ebene, wie sie etwa bei Holtschneider zu verfälschenden Ergebnissen in Bezug auf die Holocaust-Ausstellung des Imperial War Museums in London führte: „The current one-sided discourse about the Holocaust in the IWMHE is a discourse that refuses Jews normality as much as it refuses Jews agency." (Holtschneider 2011, 71) Dieser scharfe Angriff entpuppt sich als unhaltbar, sobald man dazusagt, dass sich Holtschneiders Analyse hier zur Gänze auf die Fotos konzentriert und Ausstellungstexte erklärtermaßen ignoriert. Doch diese finden sich zufällig auch auf ihren Abbildungen der Ausstellung, sodass etwa in der mit „Spiritueller Widerstand“ übertitelte Sektion völlig unmissverständlich deutlich wird, dass man trotz aller Widrigkeiten soweit wie möglich jüdische Bräuche und Erziehung fortführte, Konzerte gab, Theater spielte, Tagebuch schrieb, Zeitungen herausgab und die Verfolgung dokumentierte. (Holtschneider 2011, 67)

35 „Neue Medien“ (Hoskins und Holdsworth 2015), wie sie etwa das Jewish Museum and Tolerance Center in Moskau oder das Museum der Geschichte der polnischen Juden in Warschau prägen, sind in den hier systematisch analysierten Museen selten und bleiben auf Videos beschränkt, die passiv konsumiert oder Infoseiten, die angeklickt werden können. Erst in den letzten Jahren fügte das Haus des Terrors zu seiner ungarischsprachigen Ausstellung einen Tablet-Guide in verschiedenen Sprachen hinzu. Interaktive Fragen wie ,Wie hättest Du gehandelt?' wurden 2018 im Zeitgeschichtemuseum in Ljubljana und im Vabamu Museum der Okkupationen und der Freiheit in Tallinn eingeführt.

Ә Open Access. (C 2021 Ljiljana Radonić, publiziert von De Gruyter. (cc)BY Dieses Werk ist lizensiert unter einer Creative Commons Namensnennung 4.0 International Lizenz; Abb. ausgenommen.

https://doi.org/10.1515/9783110722055-003 


\subsection{Raum und seine Kodifizierung: Der Ort und die Rolle des Museums}

Gedenkmuseen sind ein „contested space“ (Sommer-Sieghart 2006, 159f; Hall 1997, 8; Simon 2010), und haben einen bestimmten Rang in der nationalen ,Hierarchie des Gedenkens' (Beier-de-Haan 2005, 12). Der erste Schritt bestand darin, sich einen umfassenden Einblick in die Entstehungsgeschichte, den Wandel und die Rolle des Museums im jeweiligen nationalen Kontext zu verschaffen. (Rother 2005, 258) Im Fokus der site analysis steht hier der Ort: Befindet sich das Museum in situ am Ort von Massenverbrechen (Jasenovac, Theresienstadt, Museum der Genozidopfer in Vilnius, Haus des Terrors in Budapest) oder steht es im weiteren Sinn an dem Ort des historischen Geschehens (Museum des Warschauer Aufstands, Museum des Slowakischen Nationalaufstands, Holocaust-Gedenkzentrum in Budapest)? Diente das Gebäude vorher für ein Museum mit einem anderen Zweck (Museum der Okkupation Lettlands - zuvor Museum der Roten Schützen, Zeitgeschichtemuseum in Ljubljana - zuvor Museum der Volksrevolution) oder wurde das Gebäude nach 1989 eigens für den Museumszweck errichtet (Museum der Okkupationen in Tallinn)? Der spezifische Ort prägt selbstredend das Leitnarrativ des Museums, aber in manchen Fällen ignorieren In-situ-Ausstellungen aus geschichtspolitischen Gründen bestimmte Spuren des historischen Ortes - wie ich am Beispiel des Jasenovac-Gedenkmuseums und des Museums der Genozidopfer in Vilnius zeige.

Der Ort verweist auch auf die Bedeutung der Institution in der Erinnerungskultur und Geschichtspolitik des jeweiligen Landes. (S. Offe 2000, 41) Anhand von Sekundärliteratur, der Analyse der Medienberichterstattung über die zehn Museen (wenn nötig mithilfe muttersprachlicher studentischer AssistentInnen) und der von den Museen publizierten Texte habe ich die Rolle des jeweiligen Museums in der Gesellschaft verortet. Aus politikwissenschaftlicher Sicht ist hier besonders die Rolle der Geschichtsdeutung für den gesellschaftlichen Transformationsprozess nach 1989 und die Stützung der jeweiligen politischen AkteurInnen von Interesse. Hier gibt es sehr starke Unterschiede: vom im nationalen und internationalen Kontext im Rampenlicht stehenden Haus des Terrors in Budapest, das im ersten Jahr nach seiner Eröffnung eine Million BesucherInnen verzeichnen konnte (Schmidt 2003, 17), und dem ebenfalls sehr prominent verhandelten und vielbesuchten Museum des Warschauer Aufstands, über Jasenovac, das zwar immer wieder im Fokus des (ex-)jugoslawischen „Krieges um die Erinnerung“ (Radonić 2010) steht, aber geringe BesucherInnenzahlen verzeichnet, bis hin zu Museen, die außerhalb des Landes kaum jemand kennt, wie dem Museum des Slowakischen Nationalaufstands oder dem Zeitgeschichtemuseum in Ljubljana. Mich interessiert dabei insbeson- 
dere die Frage, inwiefern das Museum vor allem für das nationale Publikum bestimmt ist oder auch bzw. primär eine internationale Signalwirkung haben soll und ob sich diese Relation mit der Zeit verändert.

Das jeweilige systematisch untersuchte Museum wird im Kontext der Geschichtspolitik und der anderen Museen des Landes, in denen ebenfalls die Zeit des Zweiten Weltkriegs thematisiert wird, analysiert. Diese Kontextualisierung umfasst jüdische Museen wie das dem Holocaust gewidmete Green House in Vilnius, das Museum der Roma-Kultur in Brno und die Pläne zur Errichtung eines Gedenkmuseums im ehemaligen ,Zigeunerlager' Lety in Tschechien, aber auch nach Beginn des Habilitationsprojekts eröffnete Museen wie das stark umkämpfte Museum des Zweiten Weltkriegs in Gdańsk oder das Museum der Geschichte der polnischen Juden in Warschau.

Die relevanten AkteurInnen identifiziere ich und analysiere ihre Rolle anhand der Museumswebseite, Publikationen des Museums sowie jenen der Museumsleitung etwa in wissenschaftlichen Sammelbänden, publizierten Interviews mit MuseumsdirektorInnen und KuratorInnen der ständigen Ausstellungen sowie der Zeitungsberichterstattung über das jeweilige Museum und verflechte dies mit der Analyse der Museumsnarrative. In der Mehrzahl der zehn Museen erfolgt der Zugriff der Politik auf das Museum direkt, etwa indem die DirektorInnen vom zuständigen Ministerium (Haus des Terrors, Holocaust-Gedenkzentrum, Jasenovac, Museum des Slowakischen Nationalaufstands) oder der Stadtregierung (Museum des Warschauer Aufstands) eingesetzt werden, im Fall von Theresienstadt nach Jahrzehnten der Marginalisierung des Gedenkens an das Ghetto ab 1991 mit der Auflage, dass es VertreterInnen der Jüdischen Gemeinde Tschechiens sein müssen. Im Fall des Hauses des Terrors und des Museums des Warschauer Aufstands ist vor allem zu betonen, dass die Häuser Aushängeschilder der jeweiligen mnemonic warriors sind: von Viktor Orbán im Zuge des ungarischen Wahlkampfs 2002 seinen WählerInnen ,geschenkt‘ bzw. vom damaligen Stadtpräsidenten Lech Kaczyński als Prestigeprojekt in Vorbereitung auf die von den Kaczyński-Zwillingen angeführte erste PiS-Regierung in Polen 2005-2007. (Borodziej 2011, 145) Das Museum der Okkupation Lettlands wurde 1993 zunächst als privat - vor allem von ExilLettInnen - finanziertes gegründet, wird seit 2006 jedoch vom Staat mitfinanziert (Museum of Occupation of Latvia, o. J.). Es steht im Dauerkonflikt mit der von russischsprachigen LettInnen angeführten Stadtregierung Rigas, was den Ausbau des Museums und die Rückkehr in die 2012 für die Renovierung verlassenen Räumlichkeiten immer wieder verzögert. Das Museum der Okkupationen in Tallinn wurde 2003 von der Kistler-Ritso-Stiftung einer exil-estnischen Familie gegründet, wird jedoch auch vom estnischen Kulturministerium kontinuierlich unterstützt. Unter den hier untersuchten Museen ist es sicher das am wenigsten an offizielle Vorgaben gebundene. Aber auch in diesem Fall gilt: „Designers of such museums are 
not the agents of entirely rationalized policy implementation processes, but unwitting spokespersons voicing the currently accepted and understandable discourse“. (Wahnich 2008, 234)

\subsection{Leitnarrativ und Storyline}

Das Leitnarrativ eines Museums fungiert als überwölbende These, während die Storyline die unterstützende Argumentationskette liefert - mithilfe von 3D-Objekten, Fotografien, Videos, Dokumenten etc. (MacDonald und Fyfe 1996) Im Fokus steht die Analyse der ständigen Ausstellungen und Museumsguides der zehn Museen und die Herausarbeitung einer Typologie der Narrative. Auch wenn auf der vorherigen Ebene die Prozesshaftigkeit von Museen und deren Dauerausstellungen sowie die Rolle verschiedener AkteurInnen und politischer Verstrickungen in den Blick genommen wurde, so gilt es hier, den „Zeigegestus“ (Muttenthaler und Wonisch 2003, 59) ernst zu nehmen. Bei der Analyse wird zwar im Hinterkopf behalten, dass Sichtbares und unsichtbar Gebliebenes unlösbar miteinander verbunden sind, dennoch wird hier die realisierte Ausstellung als ,Produkt‘ des zuvor beschriebenen Aushandlungsprozesses untersucht.

Alle zehn systematisch untersuchten Museen sowie weitere für den Kontext relevante Museen bzw. ständige Ausstellungen habe ich umfassend fotografiert (1.000-2.000 Aufnahmen pro Museum) und die Videos aufgenommen, um eine erschöpfende Analyse und Beantwortung neu auftauchender Fragen auch zu einem späteren Zeitpunkt zu ermöglichen. ${ }^{36}$

Leitfragen auf dieser Ebene der diskursanalytischen Untersuchung sind: Aus wessen Perspektive wird ,die Geschichte‘ erzählt, wessen Geschichten werden marginalisiert? Liegt der Fokus auf Massenverbrechen, Krieg, Okkupation, Widerstand oder Kollaboration? Welche Rolle spielen im Vergleich zum Narra-

36 Die einzige Ausnahme ist hier das Haus des Terrors, das eine Fotografiegenehmigung zu Forschungszwecken ohne Begründung verweigerte und mir einzig die Verwendung der auf der Museumswebseite publizierten Fotografien erlaubte. Da es sich hierbei um Überblicksaufnahmen gesamter Räume handelt und die Ausstellung als einzige bloß in der Landessprache gehalten ist, musste in diesem Fall zusammen mit einer ungarischen Assistentin in einer AudioAufnahme jedes Exponat, Foto und Video beschrieben und jeder Text für die spätere Analyse übersetzt werden. Es kann nur gemutmaßt werden, dass der Grund für diese Politik die von Anfang an anhaltende scharfe internationale Kritik an dieser Institution, des Aushängeschilds der Fideszschen Geschichtspolitik, ist. Die Ablehnung der Fotogenehmigung erfolgte im Juni 2014, also noch vor dem expliziten anti-europäischen Schwenk der Regierung Orbán, könnte aber bereits im Sinne des aktuellen autoritären Backlashs in Ungarn gedeutet werden. 
tiv der Mehrheitsbevölkerung die Erinnerungen der Minderheiten, etwa der russischsprachigen Bevölkerung im Baltikum, wenn überhaupt? Gibt es Verweise auf internationale AkteurInnen oder Vorbilder und wenn ja, auf welche Weise? Manche der Fragen tauchten erst im Vergleich der Museen auf, etwa warum zwei der drei baltischen Länder ihre Museen ,Museum der Okkupation(en)` genannt haben, während das litauische Museum von 1992 bis 2018 den Namen Museum der Genozidopfer trug, der radikaler auf die These vom ,doppelten Genozid' (Himka und Michlic 2013, 17) abzielte.

Zentral für die Analyse des Leitnarrativs sind neben den Ausstellungen auch die Museumsführer, die im Fall von sechs der zehn Museen auch eine Untersuchung des diachronen Wandels des Narrativs erlauben. Die Zahl der verfügbaren, von den Museen publizierten Führer und Ausstellungskataloge variiert stark von Museum zu Museum, je nachdem, ob es bereits in der sozialistischen Ära existiert hat oder wie oft die Ausstellung geändert wurde. Es folgt eine Übersicht der insgesamt 53 Publikationen, die einer Diskursanalyse ${ }^{37}$ (und auf der nächsten methodologischen Ebene auch einer Bildanalyse) unterzogen wurden, auch um - wo dies möglich ist - den diachronen Wandel der ständigen Ausstellungen zu erfassen. (Tab. 1) Das Leitnarrativ wird im Hinblick auf folgende Fragen untersucht: Was wird als Problem identifiziert und welche Lösungsvorschläge gibt es? Wer ist das ,wir‘ in der Erzählung, wer die ,anderen‘? Wird die Geschichte von Heldentum, Opferschaft, Kollaboration oder Täterschaft erzählt - oder eine Kombination daraus? Welche Kollektivsymbole (Link 2006, 413) werden verwendet: ,helle‘ und ,dunkle‘ historische Perioden, ,Reinheit‘ des nationalen Kollektivs, Dämonisierung des ,Anderen‘, das Telos der historischen Wahrheit? In welchem Verhältnis steht ,negatives Gedächtnis‘, also die Aufarbeitung der vom eigenen Kollektiv (mit-)verantworteten Verbrechen und der Ideologien, im Namen derer sie begangen wurden, zur (kollektiven) Selbstviktimisierung?

37 Die in der Originalsprache des jeweiligen Landes publizierten Museumsführer wurden, sofern nötig, mithilfe muttersprachlicher AssistentInnen, mit den englisch- und deutschsprachigen Versionen abgeglichen. Die Analyse bezieht sich auf die Übersetzung und die (wenigen) expliziten Abweichungen vom Original bzw. implizit mitschwingende abweichende Bedeutungen werden in die Analyse eingeflochten. Das ,Fehlen` von Gedenkmuseen in Sofia und Bukarest habe ich unter Einbeziehung jener Museen und Museumsguides untersucht, in denen der Zweite Weltkrieg vorkommt: der nationalen Geschichte- und Militärmuseen usw. 
Tab. 1: Übersicht der analysierten 53 Museumsguides aus zehn Museen - geordnet nach Museumsgründungsdatum.

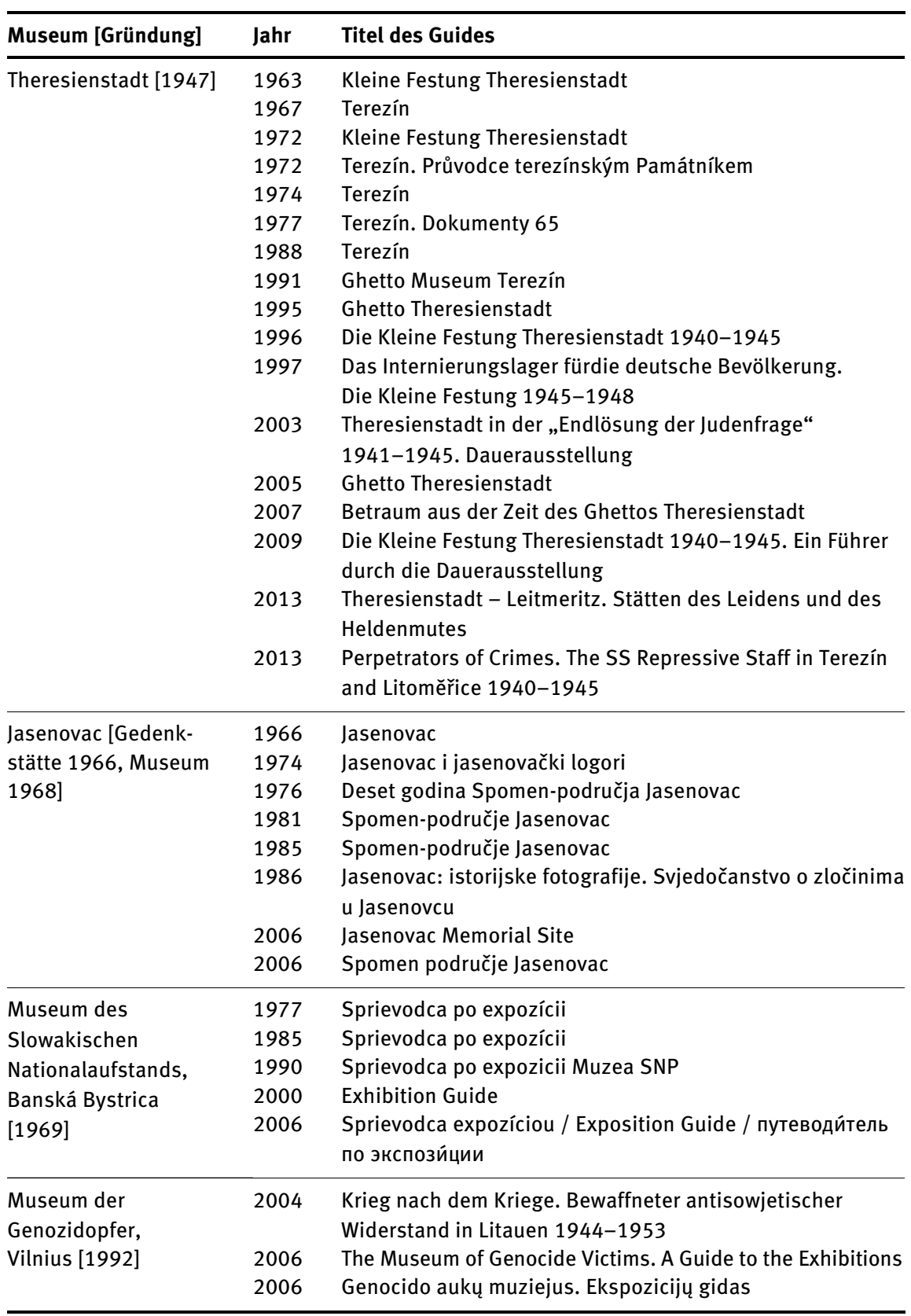


Tab. 1 (fortgesetzt)

\begin{tabular}{|c|c|c|}
\hline Museum [Gründung] & Jahr & Titel des Guides \\
\hline $\begin{array}{l}\text { Museum der } \\
\text { Okkupation Lettlands, } \\
\text { Riga [1993] }\end{array}$ & $\begin{array}{l}2008 \\
2010 \\
2017\end{array}$ & $\begin{array}{l}\text { 1940-1991. Latvia under the Rule of the Soviet Union and } \\
\text { Nationalist Socialist Germany } \\
\text { Lettland unter der Herrschaft der Sowjetunion und } \\
\text { NS-Deutschlands 1940-1991 } \\
\text { Lettland unter der Herrschaft der Sowjetunion und } \\
\text { NS-Deutschlands 1940-1991 }\end{array}$ \\
\hline $\begin{array}{l}\text { Zeitgeschichtemuseum, } \\
\text { Ljubljana [1994] }\end{array}$ & $\begin{array}{l}1998 \\
1998 \\
2007 \\
2007 \\
2009\end{array}$ & $\begin{array}{l}\text { Muzej novejše zgodovine: } 1948-98 \\
\text { Temna stran meseca: kratka zgodovina totalitarizma v } \\
\text { Sloveniji } 1945-1990 \\
\text { Rojstvo Slovenije } \\
\text { Slovenija } 1945-1960 \\
\text { The Making of Slovenia }\end{array}$ \\
\hline $\begin{array}{l}\text { Haus des Terrors, } \\
\text { Budapest [2002] }\end{array}$ & $\begin{array}{l}2003 \\
2003 \\
2008\end{array}$ & $\begin{array}{l}\text { Haus des Terrors. Andrássy Straße 30, Budapest } \\
\text { Terror Háza. Andrássy út 30, Budapest } \\
\text { House of Terror. Andrássy Street 30, Budapest }\end{array}$ \\
\hline $\begin{array}{l}\text { Museum der Okkupa- } \\
\text { tionen, Tallinn [2003] }\end{array}$ & 2014 & Estonia's Occupations Revisited. Accounts of an Era \\
\hline $\begin{array}{l}\text { Holocaust- } \\
\text { Gedenkzentrum, } \\
\text { Budapest [2004] }\end{array}$ & 2006 & $\begin{array}{l}\text { From Deprivation of Rights to Genocide. To the Memory } \\
\text { of the Victims ... } \\
\text { Jogfosztástól népirtásig }\end{array}$ \\
\hline $\begin{array}{l}\text { Museum des War- } \\
\text { schauer Aufstands } \\
{[2004]}\end{array}$ & $\begin{array}{l}2006 \\
2007 \\
2007 \\
2011 \\
2014 \\
2015\end{array}$ & $\begin{array}{l}\text { Katalog. Muzeum Powstania Warszawskiego } \\
\text { Guidebook to the Warsaw Rising Museum } \\
\text { Przewodnik po Muzeum Powstania Warszawskiego } \\
\text { Katalog. Muzeum Powstania Warszawskiego } \\
\text { Przewodnik po Muzeum Powstania Warszawskiego } \\
\text { Guidebook. Warsaw Rising Museum }\end{array}$ \\
\hline
\end{tabular}

\section{3 Ästhetik, Objekte, Visuelles und Texte im Detail}

Während für die diachrone Analyse des Wandels der Museen ,nur' Sekundärliteratur und Museumsführer herangezogen werden konnten, jedoch meist nicht die früheren Ausstellungen selbst, stehen auf dieser dritten Analyseebene die aktuellen Dauerausstellungen im Fokus. Einerseits habe ich Objekte, Fotografien, Videos und Texte als jeweils eigene Medien getrennt voneinander systematisch untersucht, andererseits Museen und Ausstellungen als hybride Medien begriffen, deren Ästhetik, Installationen und Bedeutung sich vor allem auch aus dem Zusammenspiel der verschiedenen Elemente ergibt. Wie ist das Narrativ mit dem 
Ausstellungsdesign verknüpft (Architektur, Farbe, Licht, Geräusche etc.)? Welche Vorbilder werden in der ästhetischen Gestaltung gewählt und handelt es sich um unmittelbare Übernahmen von Design und Musealisierungsstrategien oder um abgewandelte Formen? Gibt es dafür eine explizite Begründung? Welche ikonischen Symbole (Paul 2006) werden in der Ausstellung und den oben vorgestellten 53 Ausstellungsguides verwendet?

Sind Gedenk- und Informationselemente getrennt voneinander im Museum und auf dem Museumsareal zu finden oder werden historische Information und Gedenken in den Ausstellungen vermischt? Können sich die BesucherInnen also gesondert für das Gedenken entscheiden, etwa nachdem sie sich in der Ausstellung über die historischen Umstände informiert haben, oder ist man mit beiden Aspekten zugleich konfrontiert? So dominieren etwa in Jasenovac von der Decke hängende Glastafeln, auf denen in weißer Schrift Namen der KZ-Opfer festgehalten wurden, die gesamte Ausstellung (Abb. 3), während das gleiche Gedenkelement im Holocaust-Gedenkzentrum in Budapest im Anschluss an die Ausstellung im Hof der für Museumszwecke restaurierten Synagoge zu finden ist. (Abb. 4)

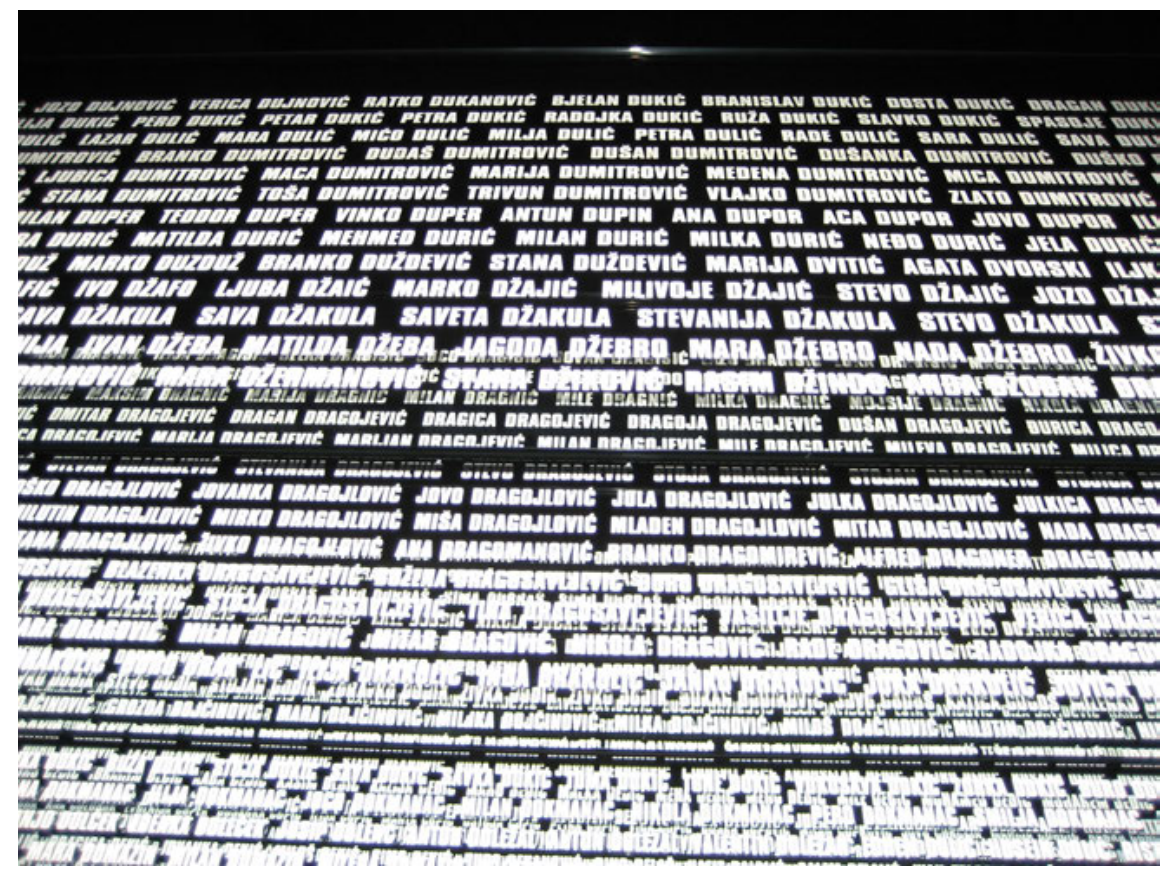

Abb. 3: Glastafeln mit Namen der Opfer in Jasenovac. 

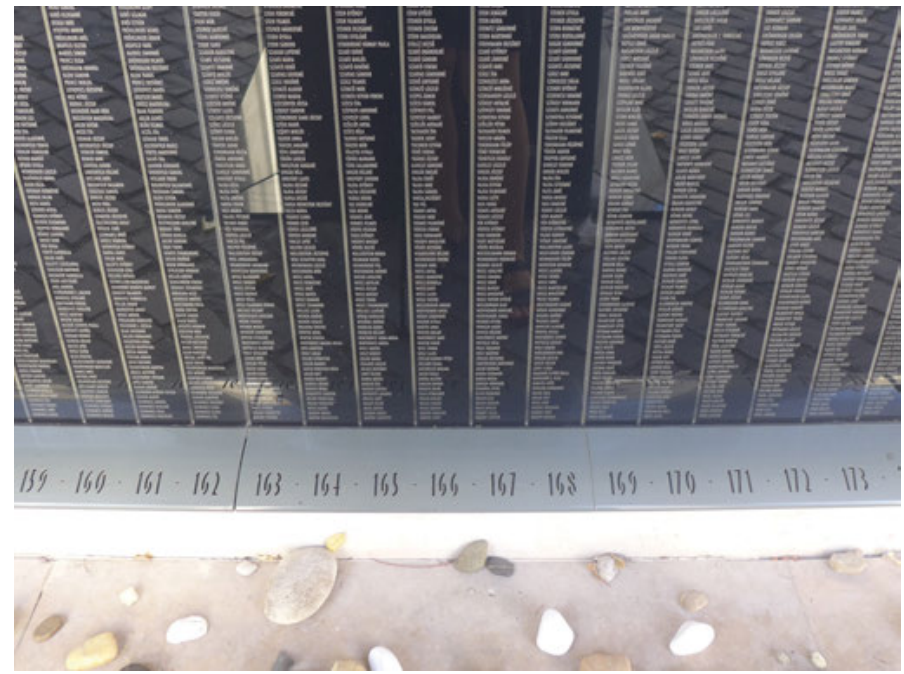

Abb. 4: Im Holocaust-Gedenkzentrum in Budapest.

Es wird ferner unterschieden zwischen verschiedenen Musealisierungstraditionen. Eine, vor allem aus den USA stammende, setzt Installationen ein, in denen die Objekte das Narrativ legitimieren sollen. Dies hat in Polen unter dem Begriff „narratives Museum“ (Majewski 2011, 152) seinen Siegeszug angetreten und dominiert auch im Haus des Terrors, das von einem Theater-Bühnenbildner inszeniert wurde. Die andere Tradition unterstellt, dass Objekte eine Aura haben, und will folglich die ,authentischen' Objekte für sich selbst sprechen lassen, wie dies vielfach in deutschen Museen der Fall ist. Vor allem im Zusammenhang von Holocaust-Ausstellungen werden eine Zyklon-B-Dose oder eine gestreifte KZ-Uniform als ikonische Symbole eingesetzt. Welche Rolle KuratorInnen den Objekten zusprechen, hat weitreichende Auswirkungen auf den Charakter der Ausstellung. In diesem Sinne können drei Arten von Ausstellungen unterschieden werden:

1. Ausstellungen mit ,ontologisch-authentischen' Objekten: Die KuratorInnen sind in diesem Fall von der „eigenständigen semantischen Aussagekraft des Objekts [...] überzeugt, vorausgesetzt es handelt sich um ein authentisches. Diese Objekte - so die Zuschreibung - tragen eine kulturelle Bedeutung ,in sich' und vermitteln diese dem/der faszinierten BetrachterIn zum Zeitpunkt ihrer Anschauung, weshalb sie nahezu ohne erläuternden Text auskommen können. Im Gegensatz dazu stellen andere MuseumsmacherInnen ebenfalls Artefakte ins Zentrum ihrer Ausstellung, beharren aber zurecht darauf, dass diese nicht ,für sich selbst sprechen' können, sondern kontex- 
tualisiert werden müssen. Die Inhalte der Ausstellung werden in beiden Fällen ausgehend von diesen ,auratischen' Objekten entwickelt.“ (Sommer-Sieghart 2006, 161)

2. Ausstellungen mit ,konstruiert-authentischen' Objekten: Objekte, die als sinnlich ansprechend eingestuft werden (Alltagsgegenstände, Archivmaterial, Fotografien, Filme, Zeitungen, Tagebücher), werden als Belege für die den BesucherInnen angebotene These herangezogen. (Korff 2002, 170) Die Unterscheidung zwischen einem ,authentischen' und einem ,nichtauthentischen' Objekt wird hier tendenziell aufgehoben: Jeder Koffer und jeder Radioempfänger aus der entsprechenden Zeit kann eingesetzt werden, um das Narrativ etwa der sowjetischen Repression zu untermauern, wie dies zum Beispiel in der 2003 im Tallinner Okkupationsmuseum installierten Ausstellung der Fall war.

3. Reine Installationen, welche die Vergangenheit als etwas inszenieren, das man berühren, riechen und durch das man wie in einer Zeitmaschine hindurchschreiten kann. (Beier-de Haan 2005, 252) Diese Form wird in der wissenschaftlichen Literatur meist kritisch gesehen. (Wahnich 2008, 228) „Statt mit unerwarteten Bezügen oder fast unmerklichen Irritationen Nachdenken und kritische Auseinandersetzung zu stimulieren, fördern sie mit der Ausrichtung auf das unmittelbare distanzlose Erlebnis lediglich Sentimentalitäten.“ (Scholze 2004, 198)

Viele Museen verwenden mehr als eine dieser Ausstellungsstrategien abwechselnd in unterschiedlichen Räumen. Im Museum des Warschauer Aufstands kann man etwa ,wie die Aufständischen' 1944 durch einen Kanal gehen oder auf den Gräbern der zerstörten Stadt Warschau wandern. Im Keller des Hauses des Terrors sollen BesucherInnen die Enge der nachgebauten Folterzellen spüren. Besonders fällt in den neueren Länderausstellungen in den nationalen Pavillons Ungarns und der Slowakei im Staatlichen Museum Auschwitz-Birkenau der Trend auf, die BesucherInnen sozusagen zu ,deportieren', sie also durch einen gläsernen, hoch-ästhetisierten Deportationswaggon hindurchschreiten zu lassen (wie im Fall der ungarischen Ausstellung) oder sie vom Geräusch eines fahrenden Zuges begleitet auf Bahnschwellen zum Deportationswaggon und aus diesem hinaustreten $\mathrm{zu}$ lassen.

Insbesondere dort, wo Objekte nicht mit einer klaren Deutung versehen sind, interessiert mich dabei die Polysemie, die Mehrdeutigkeit der Objekte. Olaf Mußmann weist darauf hin, dass der Rückzug auf die ,Aura‘ der Objekte nicht unproblematisch ist: „Kontextualisierung stellt hohe Anforderungen an MacherInnen. Gelingt sie nicht, bleiben die Exponate aus der Zeit herausgebrochene Gegenstände, isoliert, unverständlich, bedeutungslos.“ (Mußmann 2002, 
27) Das auffälligste Beispiel sind die oben erwähnten zahllosen Koffer in der 2003 im Museum der Okkupationen in Tallinn eröffneten Ausstellung (Abb. 5), die von den einen als Symbol für Exil (Mark 2008, 351), von den anderen für die Deportation der estnischen Bevölkerung in den Gulag und zugleich stark überinterpretierend als ein Versuch der Gleichsetzung mit dem Holocaust gedeutet werden: „The considerable quantity of suitcases can be read as one of the ways that the displays seek to draw subtle parallels between Nazi and Soviet society and between the Holocaust and the Gulag, not least because exhibitions of suitcases at Auschwitz and elsewhere are a long-standing symbol of the Nazi genocide.“ (Burch und Zander 2010, 61) Diese moralisch-akkusatorische Position, wonach Koffer oder Viehwaggons als Symbole der Holocaust-Erinnerung vorbehalten sein sollen, also ihr gewissermaßen ,gehören', ist nicht haltbar.

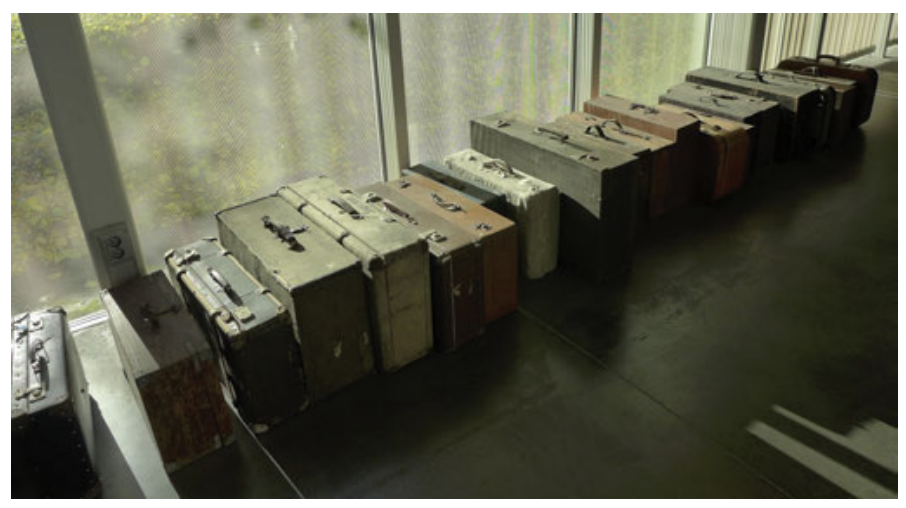

Abb. 5: Koffer im Museum der Okkupationen in Tallinn.

Gewinnbringend erwies sich die Analyse des Einsatzes von Fotografien in den Ausstellungen und der sich wandelnden Verwendung insbesondere auch in jenen Museen, die bereits seit der staatssozialistischen Ära existieren. Aus der Bildtheorie ergaben sich folgende Fragen in Bezug auf die analysierten Museen und ihre jeweilige ,Bildpolitik': Werden historische Fotografien als große Installationen und Raumteiler eingesetzt oder als historische Dokumente in der Größe der erhaltengebliebenen Abzüge? Werden - soweit bekannt - die FotografInnen genannt und die Entstehungs- und Verwendungsgeschichte des Fotos, etwa als Propagandamotiv, erwähnt? (Bring und Wegerer 2012)

Whether images are labelled with notes about their origin, purpose and history therefore allows conclusions about the curatorial intention in displaying the images and suggests distinctive messages to the viewer. The visitor's gaze is directed towards or away from the 
specific historical context of her production and original use through the use of labels, or their omission.

(Holtschneider 2011, 16)

Werden Fotografien, die TäterInnen von ihren Opfern gemacht haben (Hördler, Kreutzmüller und Bruttmann 2015) und die die Opfer stereotyp oder erniedrigend abbilden, auf andere Weise ausgestellt als private Aufnahmen der Opfer aus ihrem Leben ,davor' oder geheime Zeugnisse der Verfolgung und Vernichtung? Welche „Ikonen der Vernichtung“ (Brink 1998), also zu visuellen Symbolen gewordene Fotografien, finden sich in den hier untersuchten Museen? Werden die ,eigenen' Opfer aus der Mehrheitsbevölkerung anders visuell dargestellt als die Opfer der ,anderen'? Werden in der Ausstellung und im Museumsguide vor allem berühmte Personen und HeldInnen gezeigt oder ,normale‘ Menschen? Sollen diese ,normalen' Menschen dann ,typische‘ VertreterInnen, etwa Jüdinnen und Juden oder Romnija und Roma repräsentieren, was die Gefahr des Fortschreibens von Stereotypen birgt? Die Ausstellungen und Museumsführer wurden entlang dieser Fragen analysiert und schließlich eine postsozialistische Spezifik visueller Darstellungen in den Museen diskutiert: die Montage visueller Elemente in einer Weise, welche die Gleichheit nationalsozialistischer und staatssozialistischer Verbrechen suggeriert.

Während Diskursanalyse bereits zuvor auf der Ebene des Leitnarrativs eingesetzt wurde, wird auf dieser dritten Analyseebene darüber hinaus nach den Inhalten der Texte in den Ausstellungen und Guides im Detail gefragt. Wer ist das ,wir in der Erzählung und wie wird es charakterisiert (als Opfer, TäterInnen, KollaborateurInnen, WiderstandskämpferInnen, HeldInnen etc.)? Welche ,anderen' kommen vor und wie werden sie charakterisiert? Als zentraler Punkt stellte sich im Zuge der Analyse der zehn Museen die Frage heraus, ob ,unsere“ Opfer anders dargestellt werden als ,andere‘ Opfer. Ist von Opfern als Individuen oder etwa der Stadt Warschau oder der Nation als Kollektivopfer die Rede?

Schließlich habe ich die drei Analyseebenen verknüpft. Dies erlaubt es, unter anderem der Frage nachzugehen, ob sich erst durch die Verbindung der Analyseebenen eine ,Hierarchie der Sichtbarkeit‘ offenbart. Werden individuelle TäterInnen oder bestimmte Opfergruppen in der Ausstellung zwar thematisiert, jedoch nicht auf prominenten Texttafeln, sondern nur auf den Computerarbeitsplätzen, sodass die BesucherInnen diese Information nur dann finden, wenn sie explizit danach suchen? Gibt es ferner eine ,Hierarchie der Opfer? So betonte etwa die Ausstellung im Museum der Okkupation Lettlands (bevor sie 2012 für den Umbau aus dem Gebäude ausgezogen ist) auf der Ebene des Leitnarrativs, die Ausstellung sei nicht nur der ersten und zweiten sowjetischen Besatzung, sondern auch der NS-Besatzung gewidmet. Im Vergleich zu den beiden anderen baltischen Museen traf dies auch in stärkerem Ausmaß zu. Doch auf der Ebene 
der Analyse der Objekte und Fotografien zeigte sich, dass die über 300 ausgestellten 3D-Objekte ausschließlich den Opfern der beiden sowjetischen Besatzungen zuzuordnen waren. Der Holocaust war in der Storyline durchaus vertreten. Auf der visuellen Ebene hingegen waren die jüdischen Opfer einzig durch zwei von TäterInnen aufgenommene Fotos repräsentiert.

Die systematische Analyse auf drei methodologischen Ebenen erlaubt im nächsten Schritt einen Vergleich der Museen, ihrer Narrative und Bildpolitiken sowie ihrer unterschiedlichen Verortung im nationalen und internationalen Kontext. Haben mehrere Museen auf den ersten Blick dieselben Vorbilder, erlaubt der Vergleich die Schlussfolgerung, welche Bandbreite hier möglich ist - von der expliziten Übernahme einer bestimmten Ästhetik bis hin zu ihrer sehr freien Abwandlung mit einem inhaltlich konträren Ergebnis. Schließlich erfolgt eine Typologisierung: die Dauerausstellungen werden im Kontext der nationalen und internationalen Geschichtspolitik während der EU-Beitrittsverhandlungen und heute verortet. 